\title{
Towards a better understanding of Hipparcos
}

\author{
F. van Leeuwen \\ Institute of Astronomy, University of Cambridge, Madingley Road, Cambridge, CB3 0HA, \\ United Kingdom \\ email: fvl@ast.cam.ac.uk
}

\begin{abstract}
The formal errors on the astrometric data in the 1997 publication of the Hipparcos catalogue are, for stars brighter than about magnitude 9, largely the result of inaccuracies in the description of the along-scan attitude of the satellite. A detailed study of the dynamics of the Hipparcos satellite has led to a much improved understanding and modelling of the satellite attitude, taking into account peculiarities in the rotation of the payload (scan-phase jumps) as well as the detections of small hits. A new reduction of the Hipparcos data was initiated, in which the attitude modelling is a direct description of the dynamics of the satellite. In this so-called fully-dynamic attitude modelling the underlying torques acting on the satellite are reconstructed, and rates and error angles are obtained through integrations. Both the hits and the scan-phase jumps could be taken into account in the context of this model. The new model, including the provisions for discontinuities, led to a factor five reduction in the attitude noise. A new reduction, based on a global iterative solution like is also planned for Gaia, was started in 2004, and completed, after some 15 iterations, in 2007. In the process of this reduction, also the sensitivity of the solution to optimal connectivity between the data in the two fields of view was exposed and taken care of, as well as a couple of small-scale calibrations. The latter had not been possible to solve for in the original reductions. In the catalogue that resulted from this new reduction, the errors on the astrometric data for all but stars brighter than magnitude 4, are dominated by photon noise. Error-correlation levels in the underlying abscissa data are down by more than an order of magnitude, and play no longer any significant role. This feature very much simplifies the analysis of, for example, wide-binary stars and open cluster data.
\end{abstract}

Keywords. space vehicles, instrumentation: miscellaneous, surveys, astrometry

\section{Introduction}

The presence of a few unexpected results in the Hipparcos catalogue (ESA 1997) led to speculation concerning the overall reliability of the astrometric data contained in it, mainly based on an apparent discrepancy for the Pleiades cluster (see for example Pinsonneault et al. 1998; Pan et al. 2004; Soderblom et al. 2005). It has been verified, however, that for the individual measurements the formal errors as given in the catalogue are correct (Arenou et al. 1995; Lindegren 1995). The uncertainty in the published data is primarily caused by correlations in the underlying measurements, namely the abscissa residuals. These are derived from abscissae measurements, which were obtained from the modulated signal caused by a stellar image passing over a very regular grid in the focal plane of the telescope. This signal provided a transit time relative to the on-board clock. The along-scan attitude reconstruction provided the link between observation time and direction on the sky. This process used the same abscissa residuals that also formed input to the improvements of the astrometric parameters. The two processes use these data on very different time resolution, which allows them to be separated as part of an iterative process. This iterative process, referred to as the astrometric global iterative solution, is also planned for the Gaia data reductions (O'Mullane et al. 2006). These processes use as input the abscissa residuals, the differences between the measured and 
predicted abscissae as based on the results from the previous iteration step. Errors on the abscissa residuals become correlated for near-simultaneous measurements when there are modelling errors in the attitude reconstruction that are at least at the photon-noise level of the individual observations. Thus, bright stars are much more sensitive to such modelling errors than faint stars.

\section{Attitude modelling}

The attitude modelling for the 1997 catalogue was based on B-splines applied to the abscissa residuals as a function of time. The attitude solution was done as part of a process referred to as the great-circle reduction (van der Marel \& Petersen 1992), in which the data collected over a period of about 6 to 8 hours was projected on a reference great circle, and the local corrections for the abscissa residuals, per object, and the alongscan attitude were solved for simultaneously. One of the consequences of this procedure is that local problems in the attitude reconstruction tend to replicate with the basic angle between the two fields of view. The reason for this is that such errors affect the estimate of the abscissa residuals per object, which are in this solution at the same time incorporated in the attitude fitting at other parts of the great circle, where in turn they affect the data of yet other objects. The correlations between errors thus created are most visible for the brighter stars, and can, at separations of a few tenths of a degree, quite easily reach levels of around 0.3 and higher.

In the new reduction of the Hipparcos astrometric data (van Leeuwen 2005; van Leeuwen \& Fantino 2005; van Leeuwen 2007a,b) the attitude is modelled separately from the abscissa corrections, as a fully dynamic model (van Leeuwen \& Fantino 2003). The component modelled is the underlying torque, from which the inertial rates are obtained through integrating over the Euler equation for a free-floating rigid body in space, and the error angles through integrating over the rates. The observables, however, are not the accelerations but instead the abscissa residuals. The fitting of these residuals therefore is done as an iteration. The residuals are fitted with a fifth-order spline function, of which the second derivative provides an update to the torques model in the form of a third-order spline function. It also provides corrections to starting points for the rate and error-angle integrations. These are then used to re-calculate the abscissa residuals, and the process is repeated. The dynamical model provides a further, physical, constraint to the attitude.

\section{Peculiarities in the satellite dynamics}

Two kinds of peculiarities have been taken into account in the attitude modelling for the new reduction, hits by micro-meteorites (or space debris), and non-rigidity events. The first manifest themselves as discontinuities in the rotation rates, the second as discontinuities in the rotation phase. Both were understood to exist in the original reductions, but the possibilities to detect these events from the data were very limited. The frequency with which the scan-phase discontinuities occur was in addition much underestimated (see section 11.4 in Volume 2 of ESA 1997). In the preparations for the new reduction, some 80 hits and 1600 scan-phase jumps were identified. Once identified, they were easy to incorporate in the attitude modelling without causing significant data loss.

Scan-phase jumps happen due to small, but discrete, thermal movements of one of the three solar panels of the satellite. They can range up to 120 mas, while below 20 mas they become difficult to distinguish with certainty. To make the payload rotate by that amount, a solar panel needs to shift by a few microns. These shifts occurred as a 
result of thermal changes, mostly due to eclipses, but also due to the perigee-passage conditions. From an examination of the latter it is clear that one panel in particular was causing the problems, as most of these scan-phase jumps are concentrated around a specific rotation phase of the satellite. The rotation phase, in the way it is defined here, is equivalent to the direction of the sun as seen from the satellite. Once the jumps and hits had been identified and incorporated in the reductions, the noise-level of the along-scan attitude reconstruction dropped dramatically, by a factor four to five. Such a reduction significantly reduces he noise on the astrometric parameters for all stars brighter than about magnitude 8 to 9 .

\section{Data correlations}

The reduction in attitude noise also shows clearly in the abscissae error-correlation levels, which are reduced by more than an order of magnitude compared to the 1997 reduction, down to a level that is insignificant. This is important for the recovery of open cluster parallaxes and proper motions, as it simplifies the process and removes a major uncertainty (the actual abscissa-error-correlation levels for stars of different magnitudes) from the analysis.

To assess the accumulation of correlated errors, the scan-coincidence fraction needs to be determined. This shows what fraction of scans for a given star is shared with another star. Significant values are only found for stars at small, less than about 2 degrees, separation on the sky. Combining the error-correlation statistics with the coincidence statistics for the new reduction clearly shows that no significant level of correlated errors should be expected for the astrometric parameters of neighbouring stars. This was not the case for the 1997 catalogue. In fact, an examination of, for example, the parallax differences between the new and the old reduction shows a very significant correlation level for stars with separations less than one degree on the sky. It also shows, however, that these correlations disappear completely beyond a separation of 3 degrees. That observation is important for the Pleiades parallax determination as based on the old catalogue. Given that the cluster extends over a field of about 9 degrees diameter, the errors on the astrometric parameters of the 54 single cluster members spread over this area could only in the centre of the cluster have been significantly correlated, leaving more than half the number of stars with effectively uncorrelated errors.

\section{Connectivity}

In the construction of the new catalogue, the importance of fine-tuning the connectivity between the two fields of view became apparent. If left uncorrected, the data weight difference between the two fields of view can be considerable, more than a factor ten in some cases. The highest weights are naturally found there where bright stars are concentrated in relatively small areas on the sky, such as open cluster cores and OB associations. This issue is important for the along-scan attitude reconstruction, which provides, together with the basic-angle calibration, the linking between the two fields of view. This linking, in turn, is what makes it possible to determine absolute parallaxes from the Hipparcos data. If an area of the sky generally asserts more to much more weight than any other area it is connected to through the satellite scanning, then what may happen is that the astrometric data in that field do not get properly connected to the catalogue, and define their own local reference. This became clear during the first iteration tests for the construction of the new catalogue. Leaving the weights of the two fields uncorrected effectively meant that the new reduction was, for bright stars, mostly modelling the errors 
of the 1997 reduction. By adjusting the weight ratio between the two FoVs to a maximum value (2.7 was finally chosen), it was possible to observe how gradually in the iterations the "memory" of the old solution was lost. Thus, the new reduction should not have a connectivity problem for the astrometric data of concentrations of bright stars in small fields, such as the core of the Pleiades cluster.

\section{Transit data}

The astrometric parameters for the Hipparcos catalogue are derived from accumulated transit data. In the 1997 catalogue these transit data represented mean abscissa residuals for all field-of-view transits within the 6 to 8 hour period of observations used in the great circle reduction. All data were projected onto a reference great circle, which represents a kind of average for the instantaneous scan directions of the individual measurements. There are a couple of disadvantages in this representation, which were resolved in the new reduction.

In the new reduction the transit data are accumulated per individual field-of-view (FoV) transit, preserving the instantaneous scan direction of the transit as a basic parameter. An individual FoV transit can accidentally be affected by a superimposed image from the other FoV, but this situation will never repeat itself. Such disturbance is more difficult to eliminate after combining the transits as was done in the 1997 reductions. Individual FoV transits each have a characteristic transit ordinate, which is not exactly constant during the transit, but changes in general by no more than a few arcseconds. This allows the resolution of small-scale grid distortions as a function of transit position. Systematic residuals as a function of ordinate, and at a level of just under 1 mas, were shown to exist. These consist of two main contributions that reflect the way the grid was manufactured. The 168 by 46 scan-field prints forming the grid showed a common non-linearity and per row an average tilt. There were also small formal error drifts as a function of transit ordinate, different for the two FoVs.

Finally, the resolution of the data in the new reduction also allowed for an analysis of residual chromaticity corrections. The new reduction was able to use the epoch-colour information for very red variable stars, provided by Dimitri Pourbaix (Platais et al. 2003; Knapp et al. 2001, 2003). Thus, in general the data for red to very red stars should be much improved. Accumulation of residuals left after the astrometric-parameter determinations as a function of the colour index V - I exposed the need for residual corrections as well as small, but systematic, adjustments for the formal errors. These adjustments could easily be explained as corrections to the simplified model with which the formal errors had initially been derived. In comparison, the 1997 reductions contained a much simpler model for the chromaticity terms. All these corrections affect mainly the brighter stars, for which they improve the general reliability of both the data and the associated formal errors.

One very noticeable effect of resolving the data in FoV transits concerns double stars with separations above about 10 arcsec. In the examination of the fainter components in these systems, the exact relative position of the primary with respect to the modulating grid is essential. The scan-orientation angle was given by the local orientation of the reference great circle in the 1997 reductions, which is not accurate enough. It is given as the instantaneous scan direction per FoV transit in the new reduction. These fainter components in double systems with separations between 10 and 30 arcsec have generally very poorly determined astrometric parameters in the 1997 catalogue, but have been well resolved in the new reductions, often exposing the double system as a real physical binary 
through the similarity in proper motion and parallax with the primary component. Some 200 stars were affected this way.

\section{Stochastic solutions}

A so-called stochastic solution was implemented for objects that failed all other approaches of fitting astrometric parameters to the abscissa residuals. It was generally considered that these stochastic solutions would ultimately be resolved as due to orbital motion of double stars, but investigations by Jancart et al. (2005); Goldin \& Makarov (2007) could resolve only few as actual binaries. The 1997 catalogue contained around 1500 such cases, and of these nearly 1000 were resolved in the new reduction with simple 5 -parameter astrometric solutions. It appears that for about two thirds of these stochastic solutions the reason was an accidental accumulation of local attitude-fitting problems.

In the new solution some 500 of the old stochastic solutions were confirmed, and some 700 new ones detected. The latter is simply the consequence of the increased accuracy for the brighter stars, which also increases the sensitivity to positional disturbances.

\section{Variability-induced movers}

A variability-induced mover or VIM solution applied to variable stars with a binary component or a parasitic star positioned very close by, and of compatible brightness. Two situations can be distinguished, the companion is either the brighter or the fainter star in the system. In the first case, the duplicity effects become more noticeable when the variable star reaches maximum light, in the second case, when the variable star reaches minimum light. Correction coefficients can be introduced in the astrometric solution. These coefficients do not determine the system parameters completely, but only a combination of those: separation and a reference magnitude difference.

In trying to process these VIM detections for the 1997 catalogue, it was noticed by Pourbaix et al. (2003) that many are in fact spurious. This was confirmed in the new reduction, which showed a very low confirmation rate of VIM solutions. In particular detection for low-amplitude variables appeared to be vulnerable to anomalous detections. This was not entirely unexpected, as the detection limit for these solutions had been set to a level such that even the slightest indication from the data would be sufficient to trigger this type of solution. For the new reduction the amplitude of the variations below which no attempt was made for a VIM solution was set at 0.4 magnitudes. In addition, the detection criterion was set such that, if the distribution of the statistic is Gaussian, only about 2 per cent of the detections could be spurious. The number of detections thus obtained has dropped dramatically, to only 44 . Some of these were already known as binaries.

\section{Binary stars}

The processing of binary stars in the Hipparcos data is a very labour-intensive job, which for the new reduction has only been partly completed. Tests were carried out on the differential parameters (separation, magnitude difference and orientation) for close binaries, which showed some small-scale systematic differences that appear to be dependent on the analysis method. A small systematic difference was noted for the magnitudedifference determinations, the origin of which is not yet clear. The astrometric parameters for binary stars have been determined assuming the differential parameters as presented in the 1997 catalogue, and some further optimization is therefore still possible. The data 
required for this are included in the publication of the new reduction. The electronic publication of the data through AstroGrid will include provisions to update the catalogue with improved solutions.

\section{Formal errors and accuracy assessment}

The formal errors on the astrometric data in the 1997 catalogue were determined based on the errors assigned to the abscissa residuals, which could contain a significant contribution from the attitude reconstruction (see for details van Leeuwen 2005). Errors were furthermore adjusted in a final merging of the two independent reductions of the data, where also correlation levels between the results were established. Final corrections for error distributions lead to various adjustments of the formal errors (see further Chapter 17 of Volume 3 in ESA 1997).

In the new reduction the formal errors on the measurements were effectively fixed based on the integrated photon counts of the measurements and the relative amplitude of the first harmonic in the signal modulation. The errors are followed through the entire reduction process. The final check is obtained from the astrometric parameter fitting. Showing the residuals as a function of signal intensity still shows the same relation with the signal intensity, with only small corrections, no larger than a few per cent in most cases, of the formal errors as a function of transit ordinate and star colour (see above). The internal system of formal errors is therefore fully understood and internally consistent. It is in addition based on a single reduction, eliminating uncertainties associated with combining two sets of partly-correlated results.

The accuracy assessment of the catalogue is more difficult. A full discussion of such an assessment for the new reduction is presented by van Leeuwen (2007a). The most important, as it is relatively independent of assumptions, element in the accuracy assessment is the presence and distribution of negative parallaxes. These are the direct result of having measurement errors larger than the actual parallaxes, as will be the case for some very distant objects. Here the new reduction has created a problem. The smallest errors found on the parallaxes are just over 0.09 mas, but there are no negative parallaxes found for any of the 1000 stars with parallax errors below 0.22 mas, and for errors below 0.38 mas, only 10 negative values are found. This by itself is a clear indication that the formal errors in the new catalogue must at least be approximately right. The number of negative parallaxes is too small, and the values spread over too wide a range of actual parallax errors, to subject them to any sensible statistical test. The limited tests that could be done show that there is no proof of any additional noise on the parallaxes. Such noise could represent small distortions in the parallax-reference system, and may reflect some of the inherited problems of the 1997 catalogue. A contribution at a level of about 0.2 mas could, however, not be excluded either.

\section{Publication of the new catalogue and some preliminary results}

Background information for the construction of the new catalogue can be found in a series of papers, starting with two papers on torque analysis and attitude modelling (Fantino \& van Leeuwen 2003; van Leeuwen \& Fantino 2003). In the same volume also the orbit of the satellite was analyzed in detail (Dalla Torre \& van Leeuwen 2003), and an overview was presented of the many peculiarities that affected the Hipparcos mission (van Leeuwen \& Penston 2003). These papers were followed by a paper on the various peculiarities in the construction and data contents of the 1997 catalogue (van Leeuwen 2005) and a preview of the construction of the new catalogue (van Leeuwen \& Fantino 
2005). The catalogue has been presented on a DVD, containing also the intermediate astrometric data and various calibration files, as part of a book (van Leeuwen 2007b) describing not only how the new catalogue was derived, but also how these data can be used in astrophysical applications, such as luminosity calibrations, cluster-parameter determinations, and studies of galactic dynamics. These studies are mostly based on near-final iteration results and may therefore give slightly different values when repeated with the final catalogue. One of these studies, on the Cepheids, has already been presented (van Leeuwen et al. 2007). Several other studies are in progress. Of those still in preparation, the result on the Pleiades cluster is possibly at this moment the most interesting, as it confirms the earlier estimates that the cluster is closer to the Sun than seems to be indicated by other measurements. A partial confirmation of the earlier result was not entirely unexpected, as the cluster covers a 9 degree diameter field, and correlations in the 1997 catalogue are only effective over areas with a diameter of about two to three degrees. In addition, the position of the main sequence as defined by the new parallax measurement appears to be in good agreement with those of clusters of similar age, IC 2602 and IC 2391. Similarly, it is found that the Hyades and Preasepe isochrones coincide very well. One approach for the further interpretation of these data could be to examine the characteristics of the empirical cluster isochrones in the context of the theoretical isochrones, and see where features differ, rather than to try fitting each individual cluster to the theoretical isochrones.

\section{References}

Arenou F., Lindegren L., Froschlé M., et al., 1995, A\&BA, 304, 52

Dalla Torre A. \& van Leeuwen F., 2003, Space Sci. Rev., 108, 451

ESA (ed.) 1997, The Hipparcos and Tycho Catalogues, no. 1200 in SP, ESA

Fantino E. \& van Leeuwen F., 2003, Space Sci.Rev., 108, 499

Goldin A. \& Makarov V.V., Nov. 2007, ApJS, 173, 137

Jancart S., Jorissen A., Babusiaux C., \& Pourbaix D., Oct. 2005, A\&A, 442, 365

Knapp G., Pourbaix D., Jorissen A., 2001, A\& $A$, 371, 222

Knapp G. R., Pourbaix D., Platais I., \& Jorissen A., 2003, A\&AA, 403, 993

van Leeuwen F., Fantino E., 2003, Space Sci. Rev., 108, 537

van Leeuwen F. \& Penston M. J., 2003, Space Sci. Rev., 108, 471

Lindegren L., 1995, A\& $A$, 304, 61

van der Marel H. \& Petersen C. S., 1992, A\& A, 258, 60

O'Mullane W., Lammers U., Bailer-Jones C., et al., 2006, ArXiv Astrophysics e-prints

Pan X., Shao M., \& Kulkarni S. R., 2004, Nature, 427, 326

Pinsonneault M. H., Staufer J., Soderblom D. R., King J. R., \& Hanson R. B., 1998, ApJ, 504, 170

Platais I., Pourbaix D., Jorissen A., et al., Jan. 2003, A\& $A$, 397, 997

Pourbaix D., Platais I., Detournay S., et al., Mar. 2003, A\&SA, 399, 1167

Soderblom D.R., Nelan E., Benedict G.F., et al., Mar. 2005, AJ, 129, 1616

van Leeuwen F., Aug. 2005, A\&A A, 439, 805

van Leeuwen F., Nov. 2007a, $A \mathscr{G} A, 474,653$

van Leeuwen F., Sep. 2007b, Hipparcos, the New Reduction of the Raw Data, Springer, Dordrecht

van Leeuwen F. \& Fantino E., Aug. 2005, A\& A, 439, 791

van Leeuwen F., Feast M. W., Whitelock P. A., \& Laney C. D., Aug. 2007, MNRAS, 379, 723 